HAUSDORFF MEANS INCLUDED BETWEEN $(C, 0)$ AND $(C, 1)$

HERBERT J. GREENBERG AND H. S. WALL

In this paper we show that if $\phi(u)$ is any function of bounded variation on the interval $0 \leqq u \leqq \infty$ and $\phi(\infty)-\phi(0)=1$, then the function $\alpha(z)=\int_{0}^{\infty} d \phi(u) /(1+z u)$ is a regular moment function; and we show that when $\phi(u)$ is further restricted to be monotone then the Hausdorff mean determined by $\alpha(z)$ is included between $(C, 0)$ and $(C, 1)$. Conditions under which this mean is equivalent to $(C, 0)$ or to $(C, 1)$ are obtained which are analogous to the conditions found by Scott and Wall ${ }^{1}$ for the special case where $\phi(u) \equiv 1$ for $u \geqq 1, \phi(0)=0$. In $\S 1$ we give an elementary development of the notion of Hausdorff summability; $\S 2$ contains a proof that $\alpha(z)$ is a regular moment function; $\$ 3$ contains the above mentioned inclusion theorems; and $\$ 4$ contains examples and a discussion of some transformations of moment functions which are suggested by the earlier developments.

1. Hausdorff summability. Let $A=\left(a_{i j}\right)$ be any matrix in which $a_{i i} \neq 0$ and $a_{i j}=0$ for $j>i, i, j=0,1,2, \cdots$, and consider the system of equations

$$
\begin{array}{ll}
a_{00} q_{0} & =c_{0}\left(a_{00} p_{0}\right), \\
a_{10} q_{0}+a_{11} q_{1} & =c_{1}\left(a_{10} p_{0}+a_{11} p_{1}\right), \\
a_{20} q_{0}+a_{21} q_{1}+a_{22} q_{2} & =c_{2}\left(a_{20} p_{0}+a_{21} p_{1}+a_{22} p_{2}\right),
\end{array}
$$

These equations constitute a linear transformation of the sequence $\left\{p_{n}\right\}$ in to the sequence $\left\{q_{n}\right\}$, the transformation depending upon the matrix $A$ and the sequence $\left\{c_{n}\right\}$. If $\lim q_{n}=p$, we shall say that the sequence $\left\{p_{n}\right\}$ is $\left[A, c_{n}\right]$-summable to the limit $p$. A sequence $\left\{c_{n}\right\}$ such that $\left[A, c_{n}\right]$ sums every convergent sequence to its proper limit will be called $A$-regular. The following statements are almost obvious consequences of the above definitions:

(i) If $\left[A, c_{n}\right]$ transforms $\left\{p_{n}\right\}$ into $\left\{q_{n}\right\}$, and $\left[A, d_{n}\right]$ transforms $\left\{q_{n}\right\}$ into $\left\{r_{n}\right\}$, then $\left[A, c_{n} d_{n}\right]$ transforms $\left\{p_{n}\right\}$ into $\left\{r_{n}\right\}$.

(ii) If $\left\{c_{n}\right\},\left\{d_{n}\right\}$ are $A$-regular, then $\left[c_{n} d_{n}\right]$ is $A$-regular.

(iii) If $\left[A, c_{n}\right]$ sums $\left\{p_{n}\right\}$ to the limit $p$, then $\left[A, k c_{n}\right]$ sums $\left\{p_{n}\right\}$ to the limit $k p$.

Presented to the Society, February 28, 1942; received by the editors January 15, 1942.

${ }^{1}$ W. T. Scott and H. S. Wall, Transformation of series and sequences, Transactions of this Society, vol. 51 (1942), pp. 255-279. 
(iv) If $\left\{c_{n}\right\},\left\{d_{n}\right\}$ are $A$-regular, and $g+h=1$, then $\left\{g c_{n}+h d_{n}\right\}$ is $A$-regular.

(v) If $\left\{a_{n}\right\},\left\{b_{n}\right\}$ are $A$-regular, and $b_{n} \neq 0, n=0,1,2, \cdots$, then $\left[A, a_{n}\right] \supset\left[A, b_{n}\right]$, that is, every sequence summable $\left[A, b_{n}\right]$ is summable $\left[A, a_{n}\right]$ to the same limit, if and only if $\left\{a_{n} / b_{n}\right\}$ is $A$-regular.

Hausdorff summability ${ }^{2}$ is the particular $\left[A, c_{n}\right]$-summability obtained by taking $A=H=\left((-1)^{j} C_{i j}\right)$ where $C_{i j}=i ! / j !(i-j) !, 0 \leqq j \leqq i$; $C_{i j}=0, j>i$. In this case the equations (1.1) may be written

$$
\Delta^{m} q_{0}=c_{m} \Delta^{m} p_{0}, \quad m=0,1,2, \cdots,
$$

where $\Delta^{i} x_{j}=x_{j}-C_{i 1} x_{j+1}+C_{i 2} x_{j+2}-\cdots+(-1)^{i} C_{i i} x_{j+i}$; or, as may be readily shown:

$$
q_{m}=\sum_{n=0}^{m} C_{m n} \Delta^{m-n} c_{n} \cdot p_{n}, \quad m=0,1,2, \cdots .
$$

Inasmuch as we shall be dealing exclusively with Hausdorff summability from now on, we shall write regular instead of $H$-regular, whenever the occasion arises.

If $\left\{p_{n}\right\}$ is the sequence $1,1,1, \cdots$, then (1.2) becomes $\Delta^{m} q_{0}=0$, $m>0, \Delta^{0} q_{0}=q_{0}=c_{0}$, or $q_{m}=c_{0}, m=0,1,2, \cdots$; and if $\left\{p_{n}\right\}$ is the sequence $1,0,0, \cdots$ then $\Delta^{m} q_{0}=c_{m}, q_{m}=\Delta^{m} c_{0}, m=0,1,2, \cdots$ It follows that necessary conditions for $\left\{c_{n}\right\}$ to be regular are:

$$
\begin{aligned}
c_{0} & =1, \\
\lim _{m=\infty} \Delta^{m} c_{0} & =0 .
\end{aligned}
$$

Another necessary condition obtained by applying one of the well known conditions for the regularity of sequence transformations to (1.3) is:

$$
\sum_{n=0}^{m} C_{m n}\left|\Delta^{m-n} c_{n}\right| \leqq M, \quad m=0,1,2, \cdots,
$$

where $M$ is independent of $m$. These necessary conditions are also sufficient. ${ }^{3}$

Hausdorff showed that (c) holds if and only if $\left\{c_{n}\right\}$ is a moment sequence, that is,

$$
c_{n}=\int_{0}^{1} u^{n} d \phi(u), \quad n=0,1,2, \cdots, \phi(u) \in B V[0,1] ;
$$

${ }^{2}$ F. Hausdorff, Summationsmethoden und Momentfolgen, I and II, Mathematische Zeitschrift, vol. 9 (1921), pp. 74-109, 280-299.

${ }^{3}$ Hausdorff, loc. cit. 
and that (a), (b) are equivalent, respectively, to the conditions:

$$
\begin{aligned}
& \phi(1)-\phi(0)=1, \\
& \phi(u) \text { continuous at } u=0 .
\end{aligned}
$$

We recall that $(C, 0)$, or convergence, is the Hausdorff method $\left[H, c_{n}\right]$ where $c_{n}=1, n=0,1,2, \cdots$ Also, $(H, \alpha)=\left[H,(n+1)^{-\alpha}\right]$, $(E, r)=\left[H, r^{n}\right], 0<r<1$, are the Hölder method of order $\alpha$ and the Euler-Knopp method, respectively. The former is equivalent to $(C, \alpha)=\left[H, 1 / C_{n+\alpha, n}\right]$, the Cesàro method of order $\alpha$.

2. Proof that $\{\alpha(n)\}$ is a regular sequence. We define the function $\alpha(z)$ by the equation

$$
\alpha(z)=\int_{0}^{\infty} \frac{d \phi(u)}{1+z u}, \quad \phi(u) \in B V[0, \infty], \phi(\infty)-\phi(0)=1,
$$

and shall prove that the sequence $\{\alpha(n)\}, n=0,1,2, \ldots$, is a moment sequence. To do this, it suffices to show that $\Delta^{m} \alpha(n) \geqq 0$, $m, n=0,1,2, \cdots$, when $\phi(u)$ is monotone non-decreasing. But this is obvious from the formula

$$
\Delta^{m} \alpha(n)=\int_{0}^{\infty} \frac{u^{m} m ! d \phi(u)}{(1+n u)(1+[n+1] u) \cdots(1+[n+m] u)} .
$$

To show that $\{\alpha(n)\}$ is regular we must show that $\alpha(0)=1$, and that $\Delta^{m} \alpha(0) \rightarrow 0$ as $m \rightarrow \infty$. That $\alpha(0)=1$ follows from the hypothesis $\phi(\infty)-\phi(0)=1$. Write $\Delta^{m} \alpha(0)$ as the sum of three integrals $J_{1}, J_{2}, J_{3}$, with the limits of integration 0 to 1,1 to $k, k$ to $\infty$, respectively, $k>1$. Since the integrand

$$
1 /\left[\left(1+\frac{1}{u}\right)\left(1+\frac{1}{2 u}\right) \cdots\left(1+\frac{1}{m u}\right)\right]<1
$$

it follows that if $\phi(u)$ is monotone then $0 \leqq J_{3} \leqq \phi(\infty)-\phi(k)<\epsilon / 3$, $\epsilon>0$, if $k \geqq K_{\epsilon},\left(K=K_{\epsilon}\right.$ independent of $\left.m\right)$. If $k$ is fixed, then

$$
0 \leqq J_{2} \leqq 1 /\left[\left(1+\frac{1}{K}\right)\left(1+\frac{1}{2 K}\right) \cdots\left(1+\frac{1}{m K}\right)\right] \leqq \frac{\epsilon}{3},
$$

for all $m$ sufficiently large, inasmuch as $\prod_{n=1}^{\infty}(1+1 / n K)$ diverges to $\infty$. Also, $\lim _{m=\infty} J_{1}=0$ inasmuch as the integrand tends monotonically to 0 for each $u, 0 \leqq u \leqq 1$. Hence $\lim _{m=\infty} \Delta^{m} \alpha(0)=0$ if $\phi(u)$ is monotone. If $\phi(u) \in B V[0, \infty]$ it can be written as a linear combination of bounded monotone functions with coefficients \pm 1 , $\pm i$, and there- 
fore $\lim _{m=\infty} \Delta^{m} \alpha(0)=0$ in any case. We have completed the proof of the following theorem:

Theorem 2.1. The sequence $\{\alpha(n)\}$, where $\alpha(z)$ is given by (2.1), is a regular sequence.

3. An inclusion relation for $[H, \alpha(n)]$ when $\phi(u)$ is monotone. In the integral defining $\alpha(z)$ replace $z$ by $z+1$ and $u$ by $v /(1-v)$, and we obtain :

$$
\alpha(z+1)=\int_{0}^{1} \frac{(1-v) d \phi_{1}(v)}{1+z v}, \quad \phi_{1}(v)=\phi\left(\frac{v}{1-v}\right) .
$$

If $\phi(u)$ is monotone and $\phi(\infty)-\phi(0)=1$, then $\phi_{1}(v)$ is monotone, $0 \leqq v \leqq 1$, and $\phi_{1}(1)-\phi(0)=1$. Consequently, ${ }^{4}$ we have an expansion of the form

$$
\alpha(z+1)=\frac{g_{1}}{1}+\frac{\left(1-g_{1}\right) g_{2} z}{1}+\frac{\left(1-g_{2}\right) g_{3} z}{1}+\cdots,
$$

where $0 \leqq g_{n} \leqq 1, n=1,2,3, \cdots$, and where the continued fraction is to be terminated with the first identically vanishing partial quotient in case some $g_{n}$ is 0 or 1 . Excepting in the trivial case $g_{1}=1, \alpha(z) \equiv 1$, we then have:

$$
\begin{aligned}
\frac{g_{1}}{\alpha(z+1)} & =1+\left(1-g_{1}\right) z\left[\frac{g_{2}}{1}+\frac{\left(1-g_{2}\right) g_{3} z}{1}+\frac{\left(1-g_{3}\right) g_{4} z}{1}+\cdots\right] \\
& =1+\left(1-g_{1}\right) z \alpha^{*}(z+1),
\end{aligned}
$$

where

$$
\alpha^{*}(z+1)=\int_{0}^{1} \frac{(1-v) d \phi_{1}^{*}(v)}{1+z v},
$$

a function of the same kind as $\alpha(z+1)$. That is, $\phi_{1}^{*}(v)$ is monotone; and $\phi_{1}^{*}(1)-\phi_{1}^{*}(0)=1$, since ${ }^{5}$

$$
1=\phi_{1}(1)-\phi_{1}(0)=1-\left[1+\sum_{m=1}^{\infty} \frac{g_{1} g_{2} \cdots g_{m}}{\left(1-g_{1}\right)\left(1-g_{2}\right) \cdots\left(1-g_{m}\right)}\right]^{-1}
$$

and $\phi_{1}^{*}(1)-\phi_{1}^{*}(0)$ is equal to the same expression with the subscripts of all the $g$ 's advanced by unity.

On replacing $z$ by $z-1$ we therefore have the identity

${ }^{4} \mathrm{H}$. S. Wall, Continued fractions and totally monotone sequences, Transactions of this Society, vol. 48 (1940), pp. 165-184; p. 179 and p. 182.

${ }^{5} \mathrm{H}$. S. Wall, A class of functions bounded in the unit circle, Duke Mathematical Journal, vol. 7 (1940), pp. 146-153; p. 147. 


$$
\frac{\alpha(1)}{\alpha(z)}=1+[1-\alpha(1)](z-1) \alpha^{*}(z), \quad \alpha(1)<1,
$$

where

$$
\alpha^{*}(z)=\int_{0}^{\infty} \frac{d \phi^{*}(u)}{1+z u}, \quad \phi^{*}(u) \text { monotone, } \phi^{*}(\infty)-\phi^{*}(0)=1 .
$$

We are now prepared to prove the inclusion relation:

$$
(C, 0) \subset[H, \alpha(n)] \subset(C, 1) .
$$

The left half is of course a restatement of the regularity of $[H, \alpha(n)]$, and (3.2) is obviously true if $\alpha(n) \equiv 1$. To prove the right half when $\alpha(1)<1$ we write, by (3.1),

$$
\frac{1}{(n+1) \alpha(n)}=\frac{1}{\alpha(1)}\left[\frac{1}{n+1}+[1-\alpha(1)] \alpha^{*}(n)-2[1-\alpha(1)] \frac{\alpha^{*}(n)}{n+1}\right] .
$$

We have expressed the ratio $[1 /(n+1)]: \alpha(n)$ as a linear combination of regular sequences where the constants of combination add up to unity. Hence by (iv), (v) of $\$ 1$ it follows that $[H, \alpha(n)] \subset(C, 1)$.

If $\alpha(z)$ has a convergent Stieltjes ${ }^{6}$ continued fraction expansion $1 / a_{1}+z / a_{2}+z / a_{3}+z / a_{4}+\cdots$, where $a_{1}=1, a_{n}>0, \sum a_{n}$ diverges, then there is the relation

$$
1 / \alpha(z)=1+z a_{2}^{-1} \alpha^{*}(z),
$$

where $\alpha^{*}(z)=a_{2}\left(1 / a_{2}+z / a_{2}+z / a_{3}+z / a_{4}+\cdots\right)$, with the aid of which the inclusion $[H, \alpha(n)] \subset(C, 1)$ can be established. However, if $\sum a_{n}$ converges so that the continued fraction diverges, there is no assurance that a relation of the form (3.3) exists. The difficulty disappears as soon as one has (3.1).

We shall now proceed to obtain conditions under which one or the other of the inclusion symbols in (3.2) may be replaced by the equivalence symbol. In order to have $(C, 0) \approx[H, \alpha(n)]$ it is necessary and sufficient that $\{1 / \alpha(n)\}$ be a regular sequence. In particular, $1 / \alpha(n)$ must be bounded. Now $\lim _{n=\infty} \alpha(n)=\phi(+0)-\phi(0)$, and therefore a necessary condition for $\{1 / \alpha(n)\}$ to be regular is that $\phi(u)$ be discontinuous at $u=0$. This condition is also sufficient. For, by (3.1) we may write, if $\alpha(1)<1$ :

$$
\frac{1}{\alpha(n)}=\frac{1}{\alpha(1)}\left[1-[1-\alpha(1)] \alpha^{*}(n)+[1-\alpha(1)] \int_{0}^{\infty} \frac{d \phi^{*}(u)}{(1 / n)+u}\right] \text {. }
$$

${ }^{6}$ T. J. Stieltjes, Oeuvres, vol. 2, pp. 402-566. 
Supposing $\phi(u)$ to be discontinuous at $u=0$, so that $1 / \alpha(n)$ has a finite limit for $n=\infty$, this relation shows that $\int_{0}^{\infty} d \phi^{*}(u) / u<\infty$. Hence, if we put $\int_{0}^{t} d \phi^{*}(u) / u=\theta(t)$ we have:

$$
\frac{1}{\alpha(n)}=\frac{1}{\alpha(1)}\left[1-[1-\alpha(1)] \alpha^{*}(u)+[1-\alpha(1)][\theta(\infty)-\theta(0)]-\int_{0}^{\infty} \frac{d \theta(u)}{1+n u}\right] .
$$

On applying (iv) of $\S 1$ we therefore see that $\{1 / \alpha(n)\}$ is a regular sequence. In case $\alpha(1)=1$ so that $\alpha(n) \equiv 1$, the function $\phi(u)$ is discontinuous at $u=0$ and in this case $[H, \alpha(n)] \approx(C, 0)$.

In order to have $[H, \alpha(n)] \approx(C, 1)$ it is necessary and sufficient that $\{(n+1) \alpha(n)\}$ be a regular sequence. Since

$$
(n+1) \alpha(n)=\int_{0}^{\infty} \frac{d \phi(u)}{1+n u}+\int_{0}^{\infty} \frac{d \phi(u)}{(1 / n)+u},
$$

we see as in the preceding that $\{(n+1) \alpha(n)\}$ is regular if and only if $\int_{0}^{\infty} d \phi(u) / u<\infty$. We have completed the proof of the following theorem:

THEOREM 3.1. If $\alpha(n)=\int_{0}^{\infty} d \phi(u) /(1+n u)$ where $\phi(u)$ is monotone and $\phi(\infty)-\phi(0)=1$, then $(C, 0) \subset[H, \alpha(n)] \subset(C, 1)$; and $(C, 0)$ $\approx[H, \alpha(n)]$ if and only if $\phi(u)$ is discontinuous at $u=0$, while $[H, \alpha(n)] \approx(C, 1)$ if and only if $\int_{0}^{\infty} d \phi(u) / u<\infty$.

In case $\alpha(z)$ has a convergent Stieltjes continued fraction we have this theorem:

TheOREM 3.2. If $\alpha(z)=1 / a_{1}+z / a_{2}+z / a_{3}+\cdots, a_{1}=1, a_{n}>0, \sum a_{n}$ diverges, then $[H, \alpha(n)] \approx(C, 0)$ if and only if $\sum a_{2 n+1}$ converges; and $[H, \alpha(n)] \approx(C, 1)$ if and only if $\sum a_{2 n}$ converges.

This follows from Theorem 3.1, together with the fact that $\phi(+0)-\phi(0)=1 / \sum a_{2 n+1},{ }^{7}$ and the fact that $\int_{0}^{\infty} d \phi(u) / u<\infty$ if and only if $\sum a_{2 n}$ converges. ${ }^{8}$

In case the continued fraction for $\alpha(z)$ diverges, that is, $\sum a_{n}$ converges, then the odd approximants have one limit $\alpha_{1}(z)$ and the even approximants another limit $\alpha_{2}(z)$, one of which may equal $\alpha(z)$. In any case, $\alpha_{1}(z)$ and $\alpha_{2}(z)$ have integral representations of the form to which Theorem 2.1 applies. It is not difficult to show that $(C, 0) \approx\left[H, \alpha_{1}(n)\right]$, while $\left[H, \alpha_{2}(n)\right] \approx(C, 1)$. For ${ }^{9}$ the integrals

${ }^{7}$ Stieltjes, loc. cit., p. 510.

${ }^{8} \mathrm{H}$. S. Wall, On extended Stieltjes series, Transactions of this Society, vol. 31 (1929), pp. 771-781; p. 774.

${ }^{9}$ Stieltjes, loc. cit., p. 403. 
may be written as infinite series of the form $\sum_{i=0}^{\infty} M_{i} /\left(1+z u_{i}\right)$, $0 \leqq u_{0}<u_{1}<\cdots, M_{i}>0$, where $u_{0}=0$ in the case of $\alpha_{1}(z)$ whereas $u_{0}>0$ in the case of $\alpha_{2}(z)$. Hence it follows that $\phi(u)$ is discontinuous at $u=0$ in the case of $\alpha_{1}(z)$, while $\int_{0}^{\infty} d \phi(u) / u<\infty$ in the case of $\alpha_{2}(z)$.

4. Some transformations of moment sequences. If

$$
\alpha(z)=\int_{0}^{\infty} \frac{d \phi(u)}{1+z u}
$$

where $\phi(u)$ is monotone and $\phi(\infty)-\phi(0)=1$, then

$$
\frac{1-\alpha(z)}{c_{1} z}=\int_{0}^{\infty} \frac{\left(u d \phi(u) / c_{1}\right)}{1+z u}, \quad c_{1}=\int_{0}^{\infty} u d \phi(u),
$$

defines a method of summation which is equivalent to $(C, 1)$ inasmuch as $\int_{0}^{\infty}\left(u d \phi(u) / c_{1}\right) / u<\infty$. It is noteworthy that there are other moment functions which are not of the form considered above and which have the property that the transformation

$$
\alpha_{1}(z)=\frac{1-\alpha(z)}{c_{1} z},
$$

where $c_{1}$ is a normalizing factor such that $\alpha_{1}(0)=1$, carries a regular moment function $\alpha(z)$ into another regular moment function $\alpha_{1}(z)$ such that $\left[H, \alpha_{1}(n)\right] \approx(C, 1)$. For instance, if $\alpha(z)=(1+z)^{-k}$, $k=1,2,3, \cdots$, we find that

$$
\alpha_{1}(z)=\frac{1}{k}\left(\frac{1}{(1+z)}+\frac{1}{(1+z)^{2}}+\cdots+\frac{1}{(1+z)^{k}}\right) ;
$$

and it is not difficult to show that $\left[H, \alpha_{1}(n)\right] \approx(C, 1)$.

If $\alpha(z)=r^{z}, 0<r<1$, so that [H, $\left.\alpha(n)\right]$ is Euler-Knopp summability $(E, r)$, then

where

$$
\alpha_{1}(z)=\frac{1-r^{z}}{z \log (1 / r)}=\int_{0}^{1} u^{z} d \phi_{1}(u)
$$

$$
\phi_{1}(u)=\left\{\begin{array}{l}
0, \quad 0 \leqq u \leqq r, \\
1-(\log u / \log r), \quad r<u \leqq 1 .
\end{array}\right.
$$

Since $\phi_{1}(u)$ is obviously a regular mass function it follows that $\alpha_{1}(z)$ is a regular moment function. Although $(C, 1)$ and $(E, r)$ are not comparable methods, nevertheless $\left[H, \alpha_{1}(n)\right] \supset(C, 1)$. For,

$$
\alpha_{1}(z):(1+z)^{-1}=\int_{0}^{1} u^{z} d \phi_{2}(u)
$$


where

$$
\phi_{2}(u)=\left\{\begin{array}{l}
0, \quad 0 \leqq u<r \\
(1 / \log r)-(\log u / \log r)+1, \quad r \leqq u<1, \\
1, \quad u=1
\end{array}\right.
$$

and is a regular mass function. In this case, $(C, 1)$ does not include $\left[H, \alpha_{1}(n)\right]$. To see this, put $(1+z)^{-1}: \alpha_{1}(z)=\beta(z)=[\log (1 / r) / 2(1-r)]$ $\cdot \beta_{1}(z-1)$, where

Inasmuch as

$$
\beta_{1}(z)=\frac{2(1+z)}{(2+z)} \cdot \frac{1-r}{1-r^{z+1}}
$$

$$
\begin{aligned}
\frac{2(1+z)}{2+z} & =\int_{0}^{1} u^{z} d \theta_{1}(u), \\
\theta_{1}(u) & = \begin{cases}1, & u=1, \\
-u^{2}, & 0 \leqq u<1 ;\end{cases} \\
\frac{1-r}{1-r^{z+1}} & =\int_{0}^{1} u^{z} d \theta_{2}(u), \\
\theta_{2}(u) & =\left\{\begin{array}{ll}
1, & u=1, \\
r^{k}, & r^{k}<u<r^{k-1} ;
\end{array} \quad k=1,2,3, \cdots,\right.
\end{aligned}
$$

$\theta_{2}(u)=(1 / 2)\left[\theta_{2}(u+0)+\theta_{2}(u-0)\right]$ if $0<u<1$, we see that $\left\{\beta_{1}(n)\right\}$ is a regular sequence, being the product of regular sequences (cf. (ii) of $\$ 1$ ). By means of the composition formula ${ }^{10}$

$$
\theta(u)=\theta_{1}(u)+\int_{0}^{1} \theta_{2}(u / v) d \theta_{1}(v)
$$

we find that

$$
\begin{aligned}
\beta_{1}(z) & =\int_{0}^{1} u^{z} d \theta(u), \\
\theta(u) & =\left\{\begin{array}{l}
1, \quad u=1, \\
r^{k+1}-\left(r^{-k}-r\right) u^{2}, \quad r^{k+1}<u<r^{k} ; \quad k=0,1,2, \cdots,
\end{array}\right.
\end{aligned}
$$

where $\theta(u)=(1 / 2)[\theta(u+0)+\theta(u-0)], 0<u<1$.

If $\{\beta(n)\}$ is a regular sequence, then we must have $\beta(n)=\int_{0}^{1} u^{n} d \pi(u)$, $n=0,1,2, \cdots$, and at the same time we would have:

${ }^{10}$ H. L. Garabedian, Einar Hille and H. S. Wall, Formulations of the Hausdorff inclusion problem, Duke Mathematical Journal, vol. 8 (1941), pp. 193-213; p. 196. 
This implies that

$$
\beta(n+1)=\frac{\log (1 / r)}{2(1-r)} \int_{0}^{1} u^{n} d \theta(u), n=0,1,2, \cdots .
$$

$$
\frac{2(1-r)}{\log (1 / r)} \cdot \pi(u)=\int_{0}^{u} \frac{d \theta(t)}{t} .
$$

This is impossible, for the integral on the right does not exist. In fact:

$$
\begin{aligned}
& \int_{r^{k+1}-0}^{1} \frac{d \theta(u)}{u}=2(1-r)+2 r\left(1-r^{k+1}\right), \\
& \int_{r^{k+1}+0}^{1} \frac{d \theta(u)}{u}=2 r\left(1-r^{k+1}\right),
\end{aligned}
$$

so that $\int_{t}^{1} d \theta(u) / u$ does not have a limit as $t \rightarrow+0$. We have proved that $\{\beta(n)\}$ is not a regular sequence, and therefore $(C, 1)$ does not include $\left[H, \alpha_{1}(n)\right]$.

Another transformation which is suggested by Theorem 2.1 is

$$
\alpha_{1}(z)=\frac{c+\alpha(z)}{c+1}, \quad c>0 .
$$

If $\alpha(z)$ is as in Theorem 2.1, then $\alpha_{1}(z)$ is a function of the same kind; and since the effect of the transformation is to add a discontinuity to $\phi(u)$ at $u=0$ we must have $(C, 0) \approx\left[H, \alpha_{1}(n)\right]$.

One of the most important problems in the Hausdorff theory is the problem of recognizing whether or not a given sequence is a moment sequence. One way of contributing to this problem is to find transformations which carry moment sequences into moment sequences. It is perhaps of interest to point out that any Hausdorff transform of a moment sequence is a moment sequence. For, if $\left\{p_{n}\right\}$ is any moment sequence, and $\left[H, c_{n}\right]$ carries $\left\{p_{n}\right\}$ into $\left\{q_{n}\right\}$, then the relation (1.2) holds. Hence, inasmuch as $\left\{\Delta^{n} p_{0}\right\}$ is a moment sequence, and the product of two moment sequences is a moment sequence, it follows that $\left\{\Delta^{n} q_{0}\right\}$ is a moment sequence. Therefore $\left\{q_{n}\right\}$ is a moment sequence, as was to be proved. The sequence $\left\{q_{n}\right\}$ is regular if and only if $p_{0} c_{0}=1$ and either $c_{n} \rightarrow 0$ or $\Delta^{n} p_{0} \rightarrow 0$. For then and only then are the conditions (a), (b) of $\S 1$ satisfied by $\left\{q_{n}\right\}$. In particular, $\left\{q_{n}\right\}$ is regular if $\left\{p_{n}\right\},\left\{c_{n}\right\}$ are both regular.

As an example, let $c_{n}=1 /(n+1), p_{n}=r^{n}, 0<r<1$. Then $\left[H, q_{n}\right]$, where

$$
q_{n}=\frac{1+r+r^{2}+\cdots+r^{n}}{n+1}=\frac{1-r^{n+1}}{(n+1)(1-r)},
$$


is a regular Hausdorff mean. By $(4.1)$ we see that $\left[H, q_{n}\right] \supset(C, 1)$. It is easy to show that $(C, 1) \supset\left[H, q_{n}\right]$, thereby proving that $\left[H, q_{n}\right] \approx(C, 1)$. If $c_{n}=p_{n}=1 /(n+1)$, then

$$
q_{n}=\frac{1+2^{-1}+3^{-1}+\cdots+n^{-1}}{n+1}
$$

and $\left[H, q_{n}\right]$ is a regular Hausdorff mean. This mean does not include $(C, 1)$ inasmuch as $q_{n}:(n+1)^{-1}$ is unbounded.

NORTHWESTERN UNIVERSITY

\section{WHIRL-SIMILITUDES, EUCLIDEAN KINEMATICS, AND NON-EUCLIDEAN GEOMETRY}

\section{J. M. FELD}

1. Introduction. The geometry of whirls and whirl-motions in the plane had its origin in a paper by E. Kasner [6], ${ }^{1}$ was subsequently developed in a series of papers by Kasner and DeCicco $[3,7,8,9]$, adapted to the sphere by Strubecker [10], and to 3-space by Feld [4]. In this paper we shall, by adjoining three involutory transformations, extend Kasner's whirl-motion group $G_{6}$ to a mixed group $\Gamma_{6}$ - the complete whirl-motion group-composed of eight mutually exclusive, six-parameter families; these families will in turn be extended to seven-parameter families comprising the mixed group $\Gamma_{\Gamma}$ the complete whirl-similitude group. The principal results obtained are the extension of Kasner's $G_{6}$ and two representations of $\Gamma_{7}$ : a kinematic representation on the plane, $\$ 6$, and a representation in quasi-elliptic 3-space, \$7.

2. Slides, turns, and whirls. Let the point of an oriented lineal element $E$ have the rectangular coordinates $x, y$, and let the inclination of $E$ to the $x$-axis be the angle $\theta, 0 \leqq \theta<2 \pi$. Let $z=x+i y$, $\bar{z}=x-i y, \zeta=e^{i \theta}$. We shall call $z, \zeta$ the element coordinates of $E$ $(x, y, \theta)$, which, henceforth, shall be represented by the symbol $(z, \zeta)$.

Definitions. $A$ slide $S_{s}$ is a lineal element transformation that translates the point of each element along its line the same distance s.

Presented to the Society, April 13, 1940; received by the editors January 9, 1942.

1 The numbers in brackets refer to the bibliography at the end of the paper. 